\title{
A Connectivity-based Strategy for Roadside Units Placement in Vehicular Ad Hoc Networks
}

\author{
Hai-qing Liu ${ }^{1}$, Si-jing Ding ${ }^{1}$, Li-cai Yang ${ }^{1}$ and Tai Yang ${ }^{2}$ \\ ${ }^{1}$ School of Control Science and Engineering, Shandong University, Jinan, 250061, \\ Shandong, P. R. China \\ ${ }^{2}$ School of Information Science and Engineering, Shandong University, Jinan, \\ 250061, Shandong, P. R. China
}

*Corresponding author: yanglc@sdu.edu.cn

\begin{abstract}
In urban scenario, the imbalanced spatial distribution of traffic flow exerts an important influence on the performance of communication between vehicles and the Roadside Unit (RSU). This paper presents a strategy for RSU placement based on the road traffic characteristics, aiming at improving connectivity in vehicular ad hoc networks. To divide the coverage area of each RSU, we propose an Expansion and Coloration Algorithm (ECA). The average connectivity model for all vehicles in the network is established based on the results obtained from ECA. The RSUs placement problem is formulated as a combinatorial optimization problem, of which the objective is to maximize the average connectivity probability by searching for an optimal position combination of the given RSUs. Taking part of an actual urban road network as an example, the RSUs placement problem is calculated and the optimal placement scheme is evaluated Simulation results show that the optimal placement scheme obtained from our strategy leads to the best connectivity compared to uniform placement and hot-pot placemént.
\end{abstract}

Keywords: VANET RSUs Placement, Expansion and Coloration Algorithm, Average Connectivity Probability, Combinatorial Optimization Problem

\section{Introduction}

Vehicular Ad-hoc Network (VANET) is an instantiation of Mobile Ad-hoc Network (MANET) which consists of two kinds of components: moving vehicles and fixed Roadside Units (RSUs) It is a hybrid wireless network that supports both infrastructure-based and ad hoc communication modes. Increased performance and proliferation of wireless communication devices, coupling with communication needs caused by the rapid develoment of urban traffic, make VANET the most promising application-oriented network solution for a plethora of applications such as enabling automotive safety, traffic congestion prediction, mitigation and dissemination.

According to its setup and provision of services, VANET can be categorized into three types: Vehicle to Vehicle communication (V2V), Vehicle to RSU communication (V2R) and RSU to RSU communication (R2R). V2V and V2R are based on wireless communication technologies by the form of single-hop or multi-hop network, while R2R is in the form of wired or wireless communication. The main function of RSUs is to support messages exchange in the frequent partitioning environment of VANET. Using forwarding function of a RSU and the backbone established by R2R, connectivity of links will less degrade even 
though the topology is highly dynamic, especially when there is a long distance between the source node and the destination region. What's more, RSUs also provide access points to the Internet and to the infrastructure of other ITS applications such as transportation data collection and aggregation in traffic information system [1]. The RSU plays a crucial role in VANET and other ITS applications.

It is hard for each vehicle to be connectivity to at least one nearby RSU. There are two reasons. On one hand, compared to the whole urban road network, the coverage of each RSU is relatively small. Taking IEEE802.11p standard as an example, which is used as the groundwork for Dedicated Short Range Communications (DSRC) in ITS, the max communication range is only about $1000 \mathrm{~m}$. What's worse, wireless signal may be blocked by objects such as city buildings. As a result, the number of vehicles which are able to connect with RSU in one hop is relatively limited. A majority of vehicles should take advantage of multi-hop relaying via other vehicles to reach the coverage of an RSU. On the other band, there is an objective reality that spatial distribution of traffic flow in urban road network is imbalanced. Roads that are covered with more vehicles can form hot spot regions, while roads with less vehicles will degrade the connectivity of the multi-hop path between a vehicle and a RSU. Influenced by infrastructure cost, it is impossible to deploy a large number of RSUs. Therefore, a reasonable placement strategy of RSUs, which fully considers traffic distribution regularities, can not only maximize the network connectivity but also reduce cost.

Urban traffic flow has features of imbalances in both time and space scales, which can be analyzed in a statistical way. Considering the road characteristics, this paper presents a connectivity-based strategy for RSUs placement in situation of imbalanced traffic spatial distribution. We study the placement of roadside units by formulating it as a combinatorial optimization problem, of which the optmizat on objective is the maximum average connectivity between vehicles and their nearest RSUs. The main contributions of the paper are as follows:

(1) On condition that each vehicle establishes communication link with their nearest RSU, a coverage area division method named Expansion and Coloration Algorithm (ECA) is proposed. Using ECA, the nearest road segments that are under the coverage of a certain RSU in the 2-D vehicle network can be identified.

(2) The average connectivity mocel is built based on diverse traffic characteristics of road segments such as vehicle density and road length. Given a certain number of RSUs and some candidate locations, we formulate the RSUs placement problem as a combinational optimization problem. The optimization aims to find a placement scheme that can achieve the maximum average conpectivity for all vehicles.

(3) The proposedmodel is solved by using genetic algorithm. A simulation is also given to validate the performance of our method. Compared to the uniform placement and hot-spot placemen it yields higher connectivity.

The remainder of this paper is structured as follows. In Section 2, we introduce related work on the RSUs optimal placement research in VANET. Section 3 presents an expansion and coloration algorithm to divide the coverage area of each RSU in certain vehicular network. In Section 4, the average connectivity model and the optimization scheme are proposed. In Section 5, we present and discuss the results of a simulative evaluation in an actual city scenario road network. Finally, we make a conclusion and discuss the future work. 


\section{Related Work}

RSU is a critical component of VANET. It brings in significant improvement in the connectivity, routing and transmission delay of communications [2-4]. RSU study in VANET can be divided into three aspects: RSU configuration setting, data access scheduling and location placement. RSU configuration includes transmission power level, antenna type, back-bone network connectivity options et.al. RSU configuration research refers to the selection of optimal configuration settings based on surrounding environment, traffic density, network connection offerings and the overall cost [5]. RSU data access scheduling research mainly focuses on the design of effective scheduling algorithm for the RSU server, as well as the building and switching of the connection between vehicles and the RSU. With an optimal scheduling scheme, a RSU can better deal with the data access request, bringing in higher channel utilization and lower waiting delay $[6,7]$. The RSUs placement research aim to construct an economical yet efficient vehicular network by making an optimal location deploy scheme in the frequent partitioning network. Here we summarize the development of RSUs placement research.

The most accessible RSUs placement methods are the uniform placement and the hot-spot placement [8]. Uniform placement means that the RSUs afe placed uniformly according to the geometry of the road network. While for hot-spot placement, RSUs are placed in the regions with higher importance such as locations of heavy traffic flow, frequent occurring accidents and especially requiring stricter coverage. However, these methods cannot get an exact optimal scheme. Recently, a number of researchers and institutes have been proposed to look for the optimal placement strategies of RSU in VANET, conducting to improve the network performance, such as coverage, throughput, transpnissiondelay and connectivity in different scenarios.

Regarding the coverage problem of RSU, Dubey B B et al., in [9] propose that, compared to the corner of the intersection, the coverage w1 increase by nearly $15 \%$ if a RSU is placed in the center location, which has a signifiantimpact on dissemination capacity of VANET. Liang Y S et al., [5] inform that there is non-linear relationship between the cost and the RSU requirement, which is defined as the percentage of streets that are within the service range of RSUs. They also give a minimun cost scheme for the placement and configurations of RSUs. Rebai $\mathrm{M}$ et al. $10 \mathrm{f}$ develop an exact method for the 2-D coverage problem in hybrid VANET, which composes of both sensors and RSUs. Based on mathematical linear programming formulation, the proposed method can provide a solution for permitting an optimal placement of RSUs and sensors.

In order to maximize the throughput of VANET, Malandrino F et al., in [11] present the relationship between average per-downloader throughput and the number of access points provided by RSUs. They also get a conclusion that a density-based RSU access points placement yields performance close to the optimum. Wu T J et al., [8] present a RSUs placement model in highway-like scenario VANET. They take into account the impact of wireless interference, vehicle population distribution, and vehicle speed, and formulate the placement problem via an integer linear programming model. The strategy leads to good performance in throughput as compared to the uniform placement and the hot-spot placement.

Many achievements have been proposed to minimize transmission delay by deploying RSUs optimally. Taking into account the randomness of vehicle data traffic and the statistical variation of the disrupted communication channel, Abdrabou A and Zhuang W H [12] present an analytical framework. By employing the effective bandwidth theory and the effective capacity concept, the maximum distance between RSUs that stochastically limits the worst case packet delivery delay to a certain bound is obtained. Aslam B et al., $[13,14]$ study the 
optimization problem in context of VANET applications that depend on flow of information from vehicles to roadside units both in highway and urban environment. They present two optimization methods, analytical Binary Integer Programming (BIP) method and novel Balloon Expansion Heuristic (BEH), for the placement of RSUs to find a solution which has the minimum average response time. Lochert $\mathrm{C}$ et al., [1] propose a genetic algorithm which is able to identify good positions for static roadside units in order to cope with the highly partitioned nature of VANET. The object function of the algorithm is based on travel time savings.

There are also some literatures writing about the placement of RSUs for improving network connectivity. For example, Lee J and Kim C M [15] propose a RSUs placement scheme for vehicular telematics networks, aiming at improving connectivity and reducing the disconnection interval for the given number of roadside units, the transmission range, and the overlap ratio on a certain road network. Each intersection is considered as a potential RSU location and the optimal locations are selected based on the number of yehicle-reports. However, location report is the only parameter in the model. Other traffic parameters such as vehicle density, which will exert an important effect on connectivity, are not eonsidered.

\section{Coverage Area Division}

In this section, we divide the coverage area of each RSU. In VANET, the longer the distance between the source node and the destination nodec the more uncertain the road condition expresses, and the less reliable a communication link performs. On condition that each vehicle selects the Euclidean-nearest RSU to connect with, the RSU should cover a minimum desired area of streets. We present a method named Expansion and Coloration Algorithm (ECA) and our objective is to identify the minimum separate coverage areas of RSUs.

In ECA, each RSU has a unique color identification to represent road segments in its coverage. In order to facilitate analysis, we make discretization of road network structure by dividing all road segments equally into cells. In the 2-D space constrained by the road topology, suppose that there are boundaries around every RSU and they are capable of expanding to follow the road network. All boundaries expand independently at a same speed, for example, one cell per time step. Once a cell is traversed by a RSU boundary, it will be painted the match ing color of the RSU. All RSUs' expansion and coloration processes start at the same initiar time and vill finish when all cells in the network are colored.

Given a certain region of urban road network, let $S$ be the set of road segments in the area. Suppose there are ${ }_{R S E}$ RSUs placing in the network. Based on the assumption that a vehicle only selects the nearest RSU to connect with, we can formulate the problem as separating $S$ into $N_{R S U}$ subsets satisfying the following conditions:

$$
\text { 2 } S_{i} \cap S_{j}=\varnothing, \quad S_{1} \cup \cdots \cup S_{i} \cup \cdots \cup S_{N_{R S U}}=S,\left(i, j=1 \sim N_{R S U} ; i \neq j\right)
$$

Where $S_{i}$ denotes the road segments set covered by the $i$ th RSU. Compared to other RSUs, vehicles in these road segments are able to connect with the $i$ th RSU through the shortest route.

The notions used in ECA in this section are summarized as follows:

$t:$ time step of the algorithm; 
$U_{i}(t)$ : cells which are under the coverage of $i$ th RSU at time step $t$, when the algorithm finishes, cells in $U_{i}(t)$ will make up road segments in $S_{i}$;

$\Delta U_{i}(t)$ : new cells in $U_{i}(t)$ compared to $U_{i}(t-1)$;

$R_{i}(t)$ : neighbors of $\Delta U_{i}(t)$. If a cell is in the middle of a road, its neighbors are the two adjacent cells both upstream and downstream of the road. While a cell locates at the intersection, its neighbors are the adjacent cells on different directions. Neighbors of a cell are shown in Figure 1.
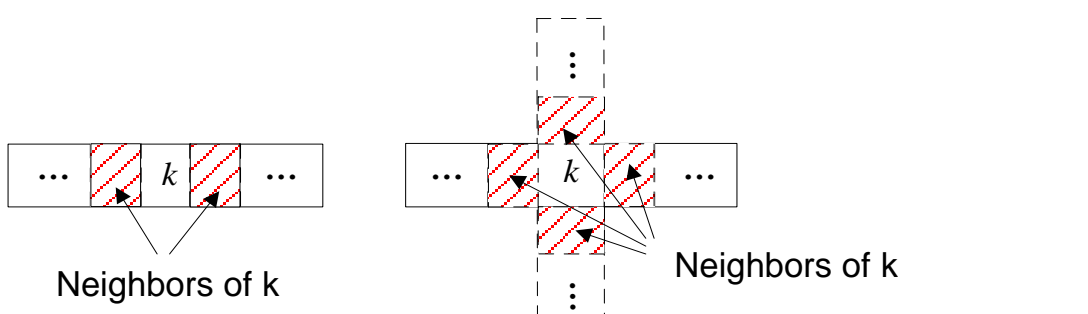

Figure 1. Neighbors of a cell

$R_{i}^{\prime}(t)$ : uncolored cells in $R_{i}(t)$;

$C_{i}$ : color identification of $i$ th RSU, coverage areas of different RSUs are represented by different colors.

At the beginning of our algorithm, at $\lambda \in 0$ time step, all cells are uncolored and $U_{i}(0)$ is the location of each RSU RSUs get thein neighbors $R_{i}(0)$ and $R_{i}^{\prime}(0)$ according to their locating cells. At $t=1$ time step expansion and coloration start. Color cells in $R_{i}^{\prime}(t) C_{i}$, meanwhile, let

$$
U_{i}(1)=U_{i}(0) \cup R_{i}^{\prime}(0), \Delta U_{i}(1)=U_{i}(1)-U_{i}(0) \text {. }
$$

Further work is to be done to identify neighbors $R_{i}(1)$ of $\Delta U_{i}(1)$ and uncolored cell $R_{i}^{\prime}(1)$ in $R_{i}(1)$. As the parameter $t$ increases, the above-mentioned process is repeated until $R_{i}^{\prime}(t)=\varnothing$, reaching the end of expansion and coloration of $i$ th RSU. At this moment, cells in $U_{i}(t)$ are all colored $C_{i}$. Cells in $U_{i}(t)$ make up the coverage area of the $i$ th RSU $S_{i}$. When all RSUs reach an end of the expansion and coloration process, the algorithm finishes. Using ECA, coverage areas division based on the shortest routes of RSUs can be achieved. The ECA for dividing coverage areas of RSUs is given in Figure 2.

Using ECA, we select part of the road network in Jinan, Shandong Province, China, which is shown in Figure 3(a), as an example to divide overage areas of RSUs. Suppose there are four RSUs in the network and the locations of them are P1, P2, P3 and P4 respectively. We simply use RSU(i) $(\mathrm{i}=1,2,3,4)$ to present the four RSUs. When all RSUs finish expansion and coloration, the coverage area of each RSU can be shown in Figure 3(b). 


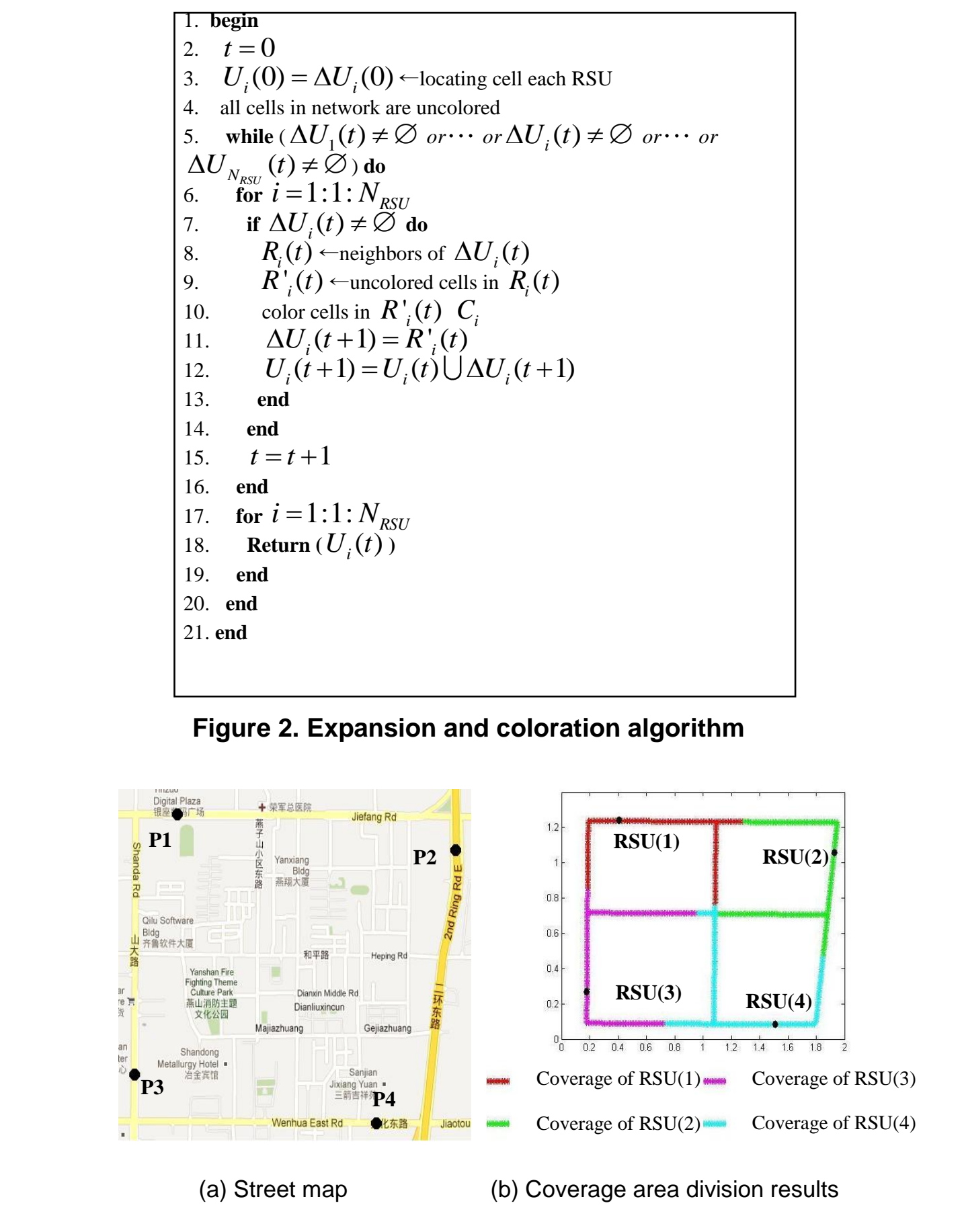

Figure 3. Coverage area division using ECA

\section{Problem Formulation}

In this section, we formulate the placement of RSUs as an optimization problem, of which the objective function is the average connectivity. The average connectivity model is established based on the statistical features of traffic flow. We study the case that traffic on 
roads is free-flow, i.e. vehicles are uniformly distributed on roads and influences caused by traffic lights are left out of consideration.

\subsection{Connectivity of road segment}

Yang Q et al., [16-18] propose a cell-based connectivity model of road segment. We use it to establish our average connectivity model. Suppose there are $n_{v e h}$ vehicles on a road segment with $n_{\text {lane }}$ lanes and $L$ length. The road segment is divided equally into $n_{\text {cell }}$ cells, the length of which is set as the average length of vehicles, for example, $d=5 \mathrm{~m}$ and $n_{\text {cell }}=L / d$. In this case, each vehicle can occupy only one cell and each cell has two states: occupied and empty. What is the probability that the distance between any two neighboring nodes is shorter than the communication range $R=n_{0} \llbracket d$, i.e. there are no more than $n_{0}$ successive empty cells on the road. The number of empty cells is $n_{\text {cell }}-\lambda_{\text {veh }}$ in one-lane scenarios, and ranges from $n_{\text {cell }}-n_{\text {veh }}$ to $n_{\text {cell }}-\left\lceil n_{\text {veh }} / n_{\text {lane }}\right\rceil$ in multi-lanes scenarios. With the assumption of uniformly distributed vehicles, the probability that the link between two sides of the road segment is connected becomes:

$$
p=1-p_{\text {dis }}=1-\sum_{k=\max \left(n_{\text {cell }}-n_{\text {veh }}, n_{0}\right.}^{\max \left(n_{\text {cell }}-\left[n_{\text {veh }} / n_{\text {lan }}\right], n_{0}\right)} p\left\{\mu\left(n_{\text {veh }}, n_{\text {cell }}\right\}=k\right\} \square\left\{\varphi\left(n_{\text {cell }}, k\right)>n_{0}\right\} .
$$

Where $p\left\{\mu\left(n_{\text {veh }}, n_{\text {cell }}\right)=k\right\}$ denotes the probability that there exists exactly $k$ empty cells. It can be calculated by formula (4) and (5).

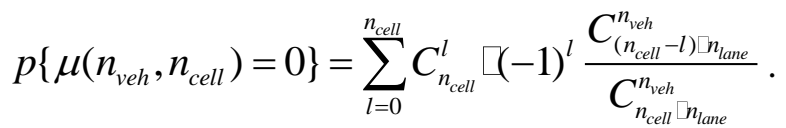

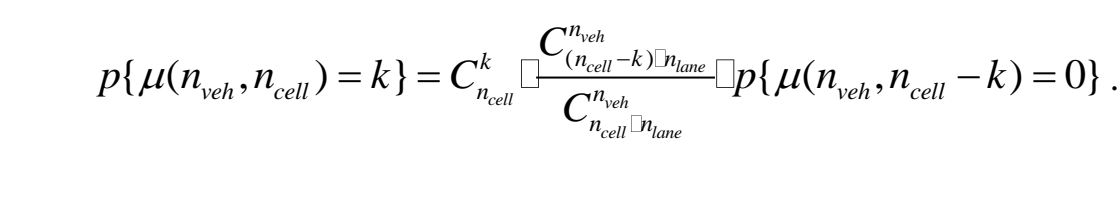

$p\left\{\varphi\left(n_{\text {cell }}, k\right)>n_{0}\right\}$ denotes the probability that there is more than successive $n_{0}$ empty cells on condition that there is empty $k$ cells on the road segment. It can be calculated by formula (6).

$$
p\left\{\varphi\left(n_{\text {cell }}, k\right)>n_{0}\right\}=1-\frac{\sum_{i=k-n_{0}}^{\min \left\{k,\left(n_{\text {cell }}-k\right)\left[n_{0}\right\}\right.} c[i]^{n_{\text {cell }}-k}}{C_{n_{\text {cell }}}^{k}} .
$$


Where the $c[i]^{t+1}$ can be calculated as follows:

$$
\left\{\begin{array}{l}
c[i]^{t+1}=\sum_{j=\max \left\{0, i-n_{0}\right\}}^{\min \left\{i, t\left[n_{0}\right\}\right.} c[j]^{t} \\
c[i]^{1}=1\left(i=0,1, \cdots, n_{0}\right)
\end{array} .\right.
$$

Given the vehicle density, together with length and number of lanes of the road segment, we can get the connectivity on the segment through the aforementioned formulas. Simply, we use a function $f$ to replace the relationship between connectivity probability and paraneters.

$$
p=f\left(n_{v e h}, n_{\text {cell }}, n_{\text {lane }}\right) \text {. }
$$

Where $n_{\text {veh }}, n_{\text {cell }}$ and $n_{\text {lane }}$ respectively represents number of vehicles, cells and lanes of a road segment.

\subsection{The average connectivity for RSUs placement}

We make an assumption that there are $N_{\text {RSU }}$ RSUs deploying in a certain region of urban road network. For the $i$ th RSU, it serves $N_{e g}(i)$ road Segments with $N_{v e h}(i)$ vehicles in the coverage area, which can be obtained using ECA. It Is important to note that road segments mentioned in our work include two different kinds: the complete road segment between two adjacent intersections, and the sub segment which is part of the complete road segment. The sum of probabilities for all vehicles connecting with the $i$ th RSU can be calculated by formula (9).

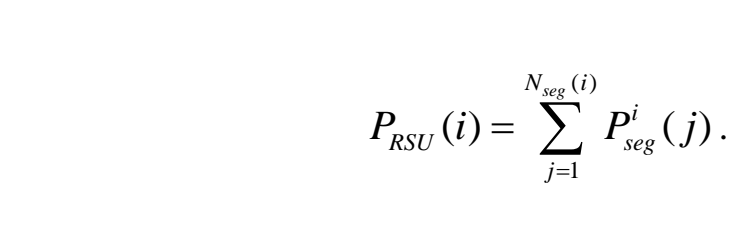

Where $P_{\text {seg }}(j)$ denotes the sum of probabilities of vehicles in the $j$ th road segment.

The average connectivity probability of all vehicles in the given road network can be calculated by formula (10).

$$
20 \bar{p}=\frac{\sum_{i=1}^{N_{R S U}} P_{R S U}(i)}{\sum_{i=1}^{N_{R S U}} N_{v e h}(i)}=\frac{\sum_{i=1}^{N_{R S U}} \sum_{j=1}^{N_{s e g}(i)} P_{s e g}^{i}(j)}{\sum_{i=1}^{N_{R S U}} N_{v e h}(i)} .
$$

Now we will analyze the solution of $P_{\text {seg }}^{i}(j)$ in different situations. As previously mentioned, $P_{\text {seg }}^{i}(j)$ denotes the sum of probabilities of vehicles in the $j$ th road segment, one 
of the road segments is served by the $i$ th RSU. According to the relative positions between the vehicle and the RSU, we classify communications into two situations: directly connecting in the case that vehicles and the RSU are in the same road segment, indirectly connecting in the case that vehicles and the RSU are distributed in different road segments.

For the $i$ th RSU, we make an assumption that the number of lanes in the $j$ th road segment is $n_{\text {lane }}^{i}(j)$, the length is $n_{\text {cell }}^{i}(j)$ and the vehicle density is $n_{v e h}^{i}(j)$. The vehicle density can be acquired according to the statistical number of vehicles in a certain period of time. We also give a stipulation that all cells in the road segment are numbered consecutively, and we define the smaller number as the upstream direction and the larger number as the downstream direction.

\section{A. Directly connecting situation}

Since wireless signal may be blocked by objects, an intersection acts as an inflection point when selecting the multiple hop transmission paths. In directly connecting situation, vehicles and the RSU are in the same road segment, passing no jnflection points. Choosing the cell where the RSU locates as the boundary point, the road segment in directly connected situation is divided into two sub-segments according to relative directions between vehicles and the RSU. The connectivity probability calculation for each sub-segment is carried on separately. Taken the scenario shown in Figure 4 as an example, of which the RSU is deployed at $y$ cell and vehicles are at the upstream direction of the RSU, we-present the solution of $P_{\text {seg }}^{i}(j)$.

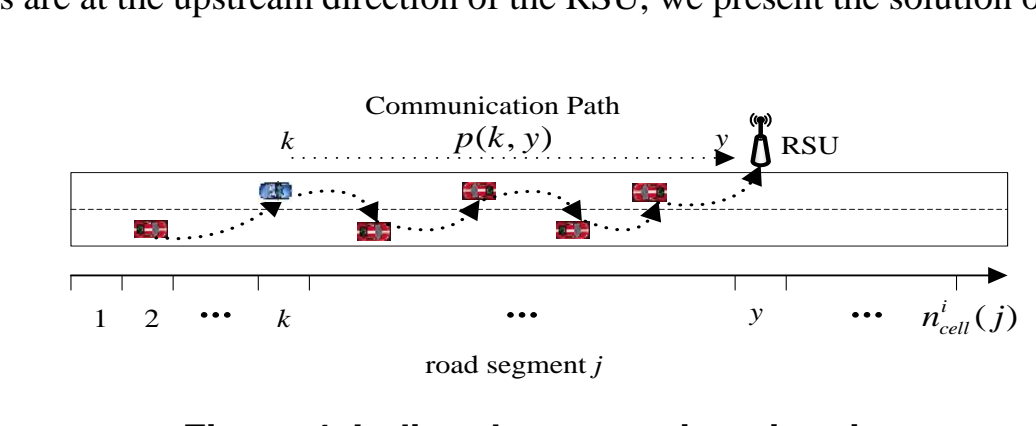

Figures. Indirectly connecting situation

Regarding vehicles at $k$ cell in the segment, the communication path is established on the sub-segment from $k$ cell to $y$ cell. Based on the precondition that vehicles are uniformly distributed on the road, parameters of the sub-segment $k \leftrightarrow y$ can be acquired as follows:

Number Of lânes: $n_{\text {lane }}^{j}(k, y)=n_{\text {lane }}^{i}(j)$.

Number of cells: $n_{\text {cell }}^{j}(k, y)= \begin{cases}y-k & \text { Vehicles are at the upstream direction of the RSU } \\ k-y & \text { Vehicles are at the downstream direction of the RSU }\end{cases}$

Vehicle density: $n_{v e h}^{j}(k, y)=\left\lceil n_{v e h}^{i}(j) \frac{n_{\text {cell }}^{j}(k, y)}{n_{\text {cell }}^{i}(j)}\right\rceil$. 
According to formula (8), the connectivity probability of data transmitting between vehicles at $k$ cell and the RSU at $y$ cell can be calculated by formula (11).

$$
p(k, y)=f\left(n_{\text {veh }}^{j}(k, y), n_{\text {cell }}^{j}(k, y), n_{\text {lane }}^{j}(k, y)\right) .
$$

Since vehicles are uniformly distributed on the road, the expected number of vehicles which occupy the $k$ th cell is $n_{v e h}^{i}(j) / n_{\text {cell }}^{i}(j)$, and different cells have the same expected number of vehicles.

$$
\bar{n}_{\text {veh }}^{j}(1)=\cdots=\bar{n}_{\text {veh }}^{j}(k)=\cdots=\bar{n}_{\text {veh }}^{j}\left(n_{\text {cell }}^{i}(j)\right)=\frac{n_{\text {veh }}^{i}(j)}{n_{\text {cell }}^{i}(j)}
$$

Therefore, the sum of probabilities for all vehicles in the $j$ th road segment connecting with the RSU can be calculated as:

$$
\begin{aligned}
P_{\text {seg }}^{i}(j) & =\sum_{k=\min (t, y)}^{k=\max (t, y)} n_{\text {veh }}^{j}(k) \square p(k, y) \\
& =\sum_{k=\min (t, y)}^{k=\max (t, y)} \frac{n_{\text {veh }}^{i}(j)}{n_{\text {cell }}^{i}(j)} \square\left(n_{\text {eh }}^{\top}(k, y), n_{\text {cell }}^{j}(k, y), n_{\text {lane }}^{j}(k, y)\right)
\end{aligned}
$$

Where $t$ denotes the farthest cell ayay from the RSU in the segment. Specially, if the segment is completely covered by the RSU, $C$ is equaled to 1 when the path passes the upstream direction of the RSU and $n_{\text {cell }}^{i}(j)$ when the path passes the downstream direction.

\section{B. Indirectly connecting situation}

As is shown in Figure 5, communication path in indirectly connecting situation consists of at least two road segments. It may pass one or more inflection points. In this situation, two road segments are containedin the path definitely, that are the segment where the source vehicles locate and the segment where the RSU locates. The connectivity probability can be calculated using the product of the connectivity probabilities of all segments in the path.

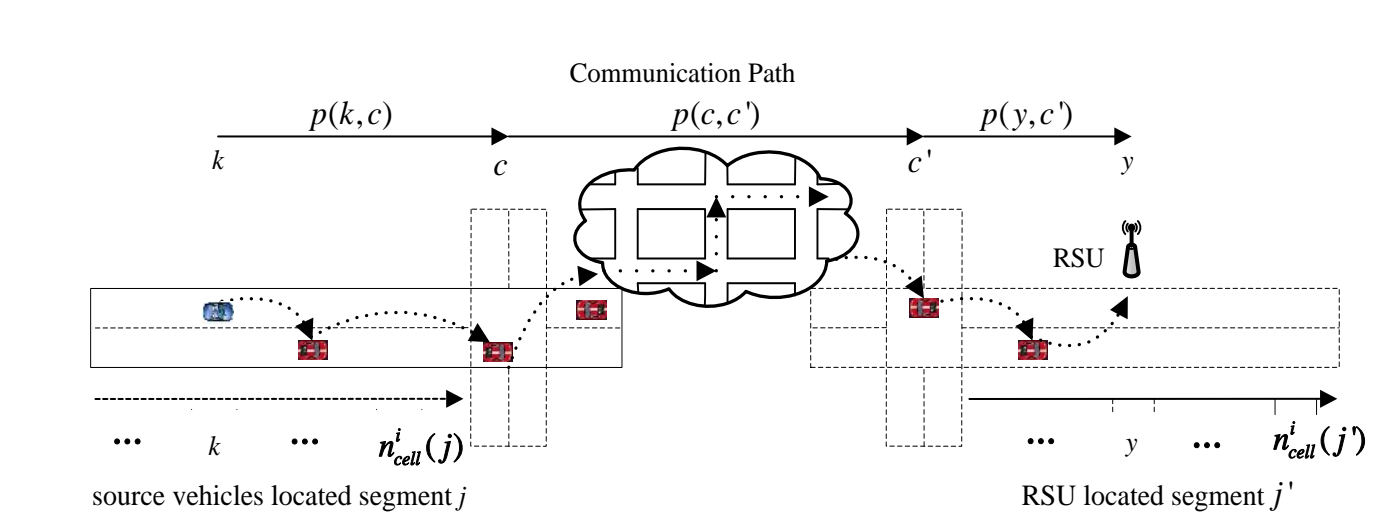

Figure 5. Indirectly connecting situation 
Suppose the source vehicles are located at the $k$ th cell of road segment $j$, and the RSU is located at the $y$ th cell of road segment $j^{\prime}$, connectivity probability of the communication path can be calculated by formula (14).

$$
p(k, y)=p(k, c) \square p\left(c, c^{\prime}\right) \square p\left(c^{\prime}, y\right)
$$

Where $p\left(c, c^{\prime}\right)$ denotes the probability between $c$ and $c^{\prime}$, and it is equaled to 1 if the communication path consists only two road segments. $p(k, c)$ denotes the probability of the source vehicles located segment and $c$ represents the position of the inflection point in the same segment. $p\left(c^{\prime}, y\right)$ denotes the probability of the RSU located segment and $c^{\prime}$ represents the position of the inflection point in the same segment. Without loss of generality, the parameters $c$ and $c^{\prime}$ are equaled to 0 when the path passes the upstream direction of the segments and equaled to $n_{\text {cell }}^{i}(j)$ and $n_{\text {cell }}^{i}\left(j^{\prime}\right)$ respectively when the path passes the downstream direction of the segments. To get $p(k, y)$, we analyze the calculation of the three probabilities.

Since segments between $c$ and $c^{\prime}$ are complete road segments and parameters of them are constant respectively. The value of $p\left(c, c^{\prime}\right)$ is also a constant, which is equaled to the product of the connectivity probabilities of all the segments calculated by formula (8).

Setting the parameter $k$ in formula (19) $c^{\prime}$, we canget the value of $p\left(c^{\prime}, y\right)$, which is a constant and shown as formula (15).

$$
p\left(c^{\prime}, y\right)=f\left(n_{\text {ven }}^{j^{\prime}}\left(c^{\prime}, C_{\text {cell }}^{\prime}\left(c^{\prime}, y\right), n_{\text {lane }}^{j^{\prime}}\left(c^{\prime}, y\right)\right)\right.
$$

Setting the parameter $k$ in formula (11) to $c$, parameters of the sub-segment $k \leftrightarrow c$ in the source vehicles located segment can be acquired as follows:

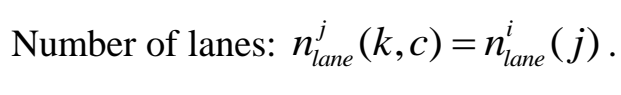

Number of cells: $n_{\text {cell }}^{j}(\hat{k}, c)= \begin{cases}k & \text { Path passes the upstream direction of the segment } \\ n_{\text {cell }}^{i}(j)-k & \text { Path passes the downstream direction of the segment }\end{cases}$ Vehicledensity: $n_{\text {veh }}^{j}(k, c)=\left\lceil n_{\text {veh }}^{i}(j) \frac{n_{\text {cell }}^{j}(k, c)}{n_{\text {cell }}^{i}(j)}\right\rceil$.

According to formula (8), the connectivity probability of data transmitting between vehicles at $k$ cell and the infection point $c$ can be calculated by formula (16).

$$
p(k, c)=f\left(n_{\text {veh }}^{j}(k, c), n_{\text {cell }}^{j}(k, c), n_{\text {lane }}^{j}(k, c)\right) .
$$


In road segment $j$, the expected number of vehicles which occupy the $k$ th cell is calculated by formula (12). Therefore, the sum of probabilities for all vehicles in the $j$ th road segment connecting with the RSU can be calculated as:

$$
\begin{aligned}
P_{\text {seg }}^{i}(j) & =\sum_{k=\min (t, c)}^{k=\max (t, c)} \bar{n}_{\text {veh }}^{j}(k) \square p(k, y) \\
& =\sum_{k=\min (t, c)}^{k=\max (t, c)} \frac{n_{v e h}^{i}(j)}{n_{\text {cell }}^{i}(j)} \square p(k, c) \square p\left(c, c^{\prime}\right) \square p\left(c^{\prime}, y\right)
\end{aligned}
$$

Where $t$ denotes the farthest cell away from the RSU in segment $j$.

\subsection{The optimization of RSUs placement problem}

Our objective is to give a RSUs placement scheme so that vehicles in the network have a maximum average connectivity probability $\bar{p}$ when connecting with their/nearest RSUs. Realizing that there are $N$ complete road segments in the region of interest and $N_{R S U}$ RSUs are going to be placed, we formulate the RSUs placement problem as a combinatorial optimization problem in the two-dimensional space.

The location of a RSU can be represented using 2-D coordinates $(x, y)$, which indicate that the RSU is deployed at the $y$ th cell of the $x$ th road segment. Each cell along a road segment will be a possible position for RSU. Therefore, there will be $\sum_{x=1}^{N} n_{\text {cell }}(x)$ possible positions in the road network, where $n_{\text {cell }}(x)$ denotes the number of cells of road segment $x$. A combination of the positions of all the $N_{R S U}$ RSUs forms a specific placement mode, which can be represented as:

$$
S_{i}=\left\{\left(x_{1}, y_{1}\right),\left(x_{2}, y_{2}\right), \cdots,\left(x_{N_{R S U}}, y_{N_{R S U}}\right)\right\}
$$

The constraints of formula (18) are as follows:

(1) $\leq x_{m} \leq N, 1 \leq x_{m}<n_{\text {cell }}\left(x_{m}\right)$;

(2) $\left(x_{m}, y_{m}\right),\left(x_{n}, y_{n}\right) \in s_{i}$, the value of $x_{m}$ and $x_{n}, y_{m}$ and $y_{n}$ are not equal simultaneousy.

Serecting $N_{R S U}$ positions from all the cells to place RSUs is a combination problem. So the number of possible combinations is:

$$
M=C_{m=1}^{N_{R S U}} \sum_{m=l l}^{N} n_{m}\left(x_{m}\right)
$$


Given a certain RSUs placement mode $s_{i}$, there is a unique coverage area division scheme using ECA and a corresponding value of the average connectivity probability $\bar{p}$. We simply use the notation $R$ for the relationship between $\bar{p}$ and $s_{i}$.

$$
\bar{p}=R\left(s_{i}\right)
$$

All possible $s_{i}$ constitute a feasible solution space $\Omega=\left\{s_{1}, s_{2}, \cdots, s_{M}\right\}$. The optimization of RSUs placement problem can be formulated as finding an optimal solution $s^{*}$ from $\Omega$, and satisfying the following condition simultaneously:

$$
\forall s_{i} \in \Omega, \bar{p}=R\left(s^{*}\right)=\max R\left(s_{i}\right)
$$

The RSUs placement problem is a combinatorial optimization problem, it is also a NPcomplete problem. Some intelligence algorithms can be used to solve it. In this paper, we use Genetic Algorithm (GA) to obtain an optimization RSUs placement scheme. The solution procedure is shown in Figure 6.

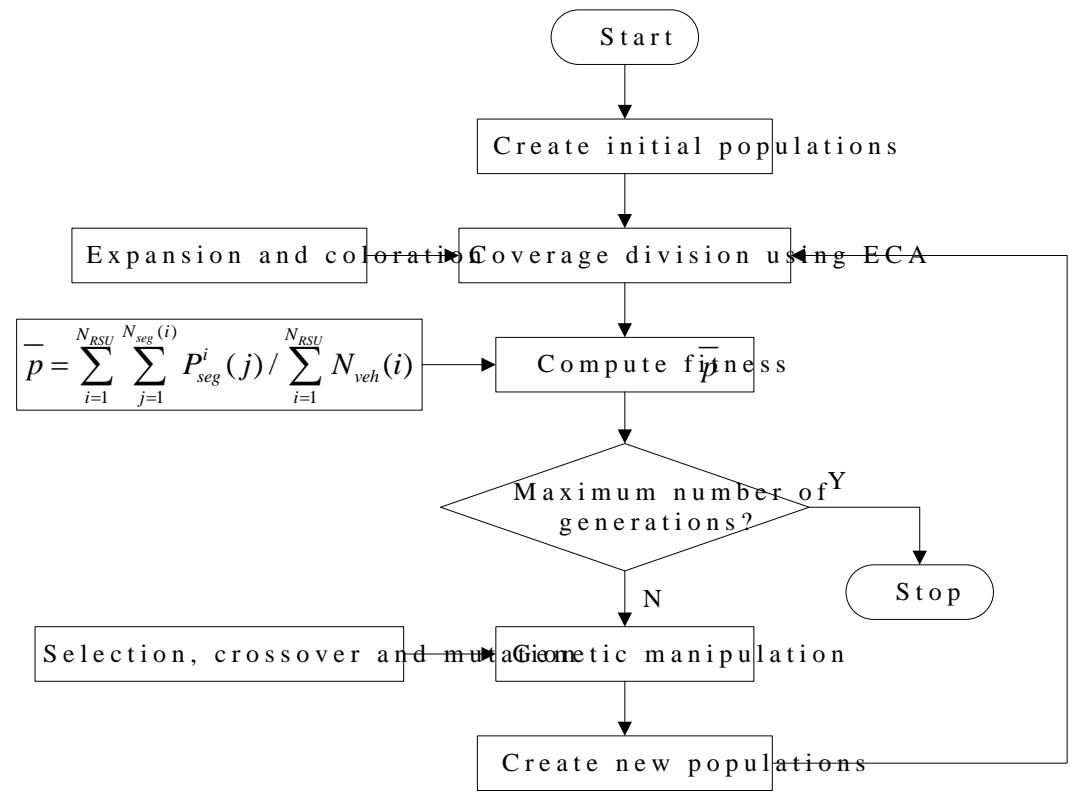

Figure 6. Solution procedure of RSUs placement problem

\section{Case Study}

In this section, we give the calculated results of our algorithm and evaluate the performance of the proposed optimization strategy.

Our simulation is based on the aforementioned urban region shown in Figure 3(a) and the topology of which is shown in Figure 7(a). It has 9 intersections and 12 road segments. To get an imbalanced traffic flow, we set the segments 1,2,5 and 10 to be four-lanes bi-directionally while others to be two-lanes. The four-lanes road segments form communication hot spots since there are more vehicles compared to others. 
The length of a cell in a road segment is divided per 5 meters, which is really a small value. For this reason, there will be a large number of cells in the road network. Selecting a certain number of locations from all these cells is tremendous work. Meanwhile, actually, there is no essential difference for the performance improvement when a RSU is placed in the two cells which are very close. In order that the RSUs placement problem can be simplified, some special cells are selected as candidate positions, for example, the inflection points or cells in middle of segments. In our simulation, 21 positions are selected as the candidate positions for placing RSUs, which are shown in Figure 7(b).

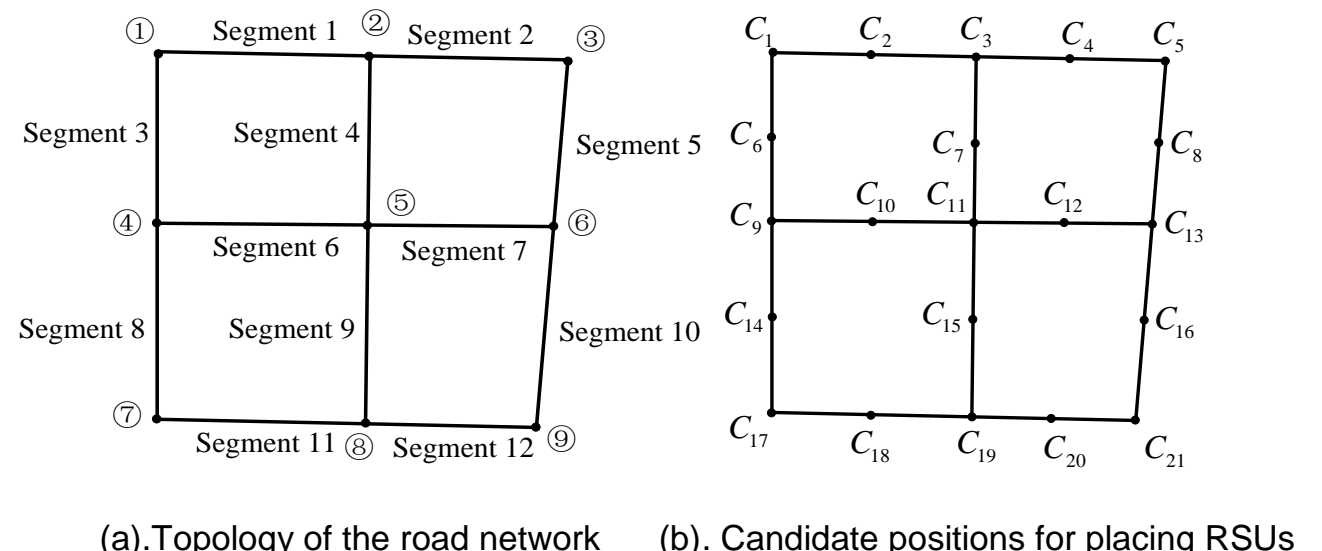

Figure 7. Topology of the road network

Other parameters used in our simulation are list in Table 1.

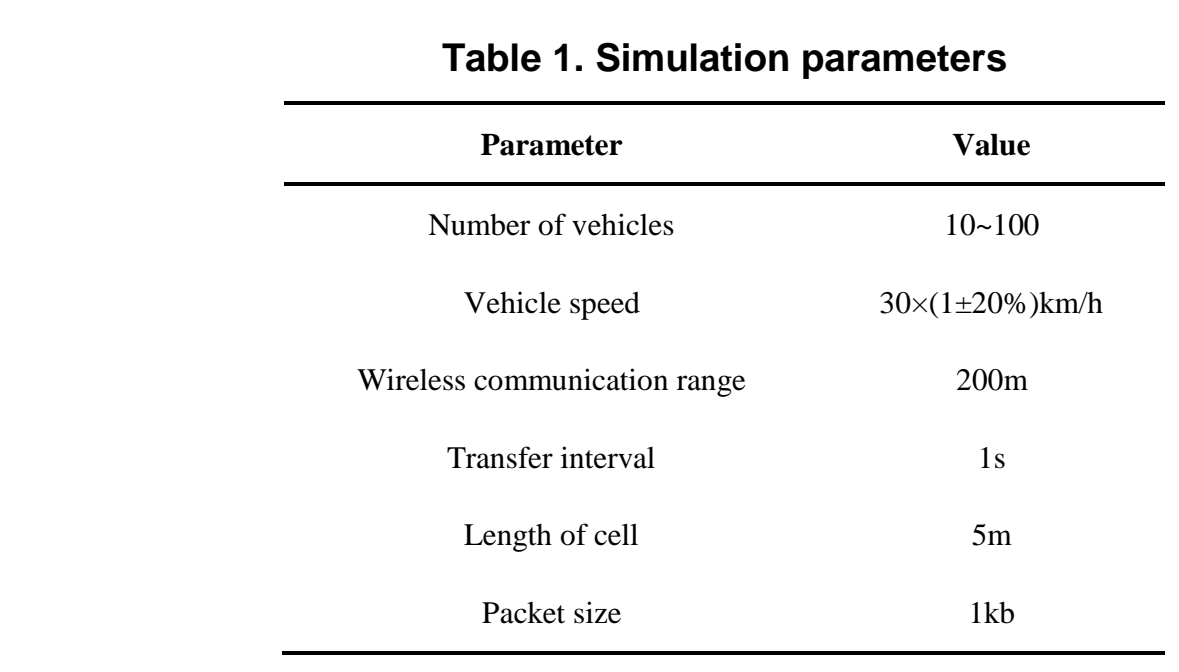

In order to obtain vehicle density in each road segment, the scenario is first simulated by VISSIM, an integrated microscopic traffic-emissions simulation platform. As long as vehicles are uniformly distributed, statistical number of vehicles in each segment is acquired using VISSIM if a certain number of vehicles are given in the network.

Four RSUs will be placed in the given network. Using the average connectivity model, we calculate the optimal placement schemes under different number of vehicles. The uniform placement scheme (RSUs are placed on C3, C6, C13 and C19) and the hot-spot placement 
scheme (RSUs are placed on C2, C4, C8 and C16) are compared to the optimal scheme. The calculated results are shown in Figure 8 and the optimal placement schemes under different vehicles are shown in Table 2.

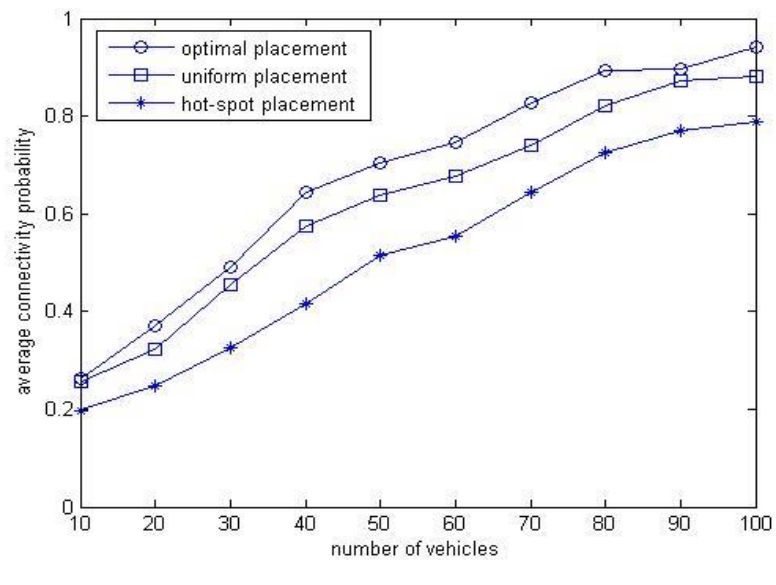

Figure 8. Calculated results

Table 2. The optimal placement schemes under different numbers of

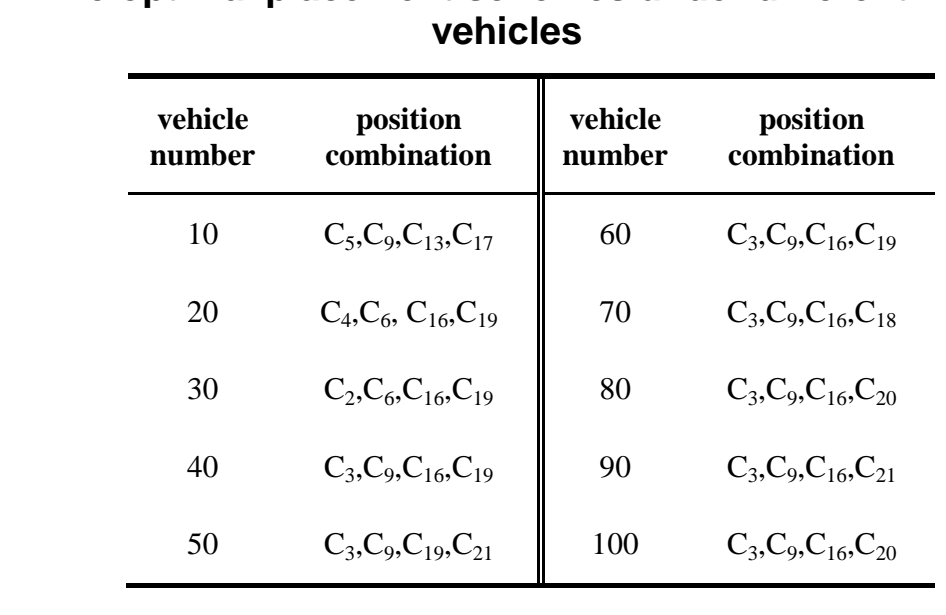

From the results shown in Figure 8, we can find that the average connectivity probability increases as the growth of vehicles. Meanwhile, both the uniform placement and the hot-spot placement cannot reach the best connectivity in our simulation network. The explanation of this situation can be summarized as follows: On one hand, the road network topology and the traffic flow distribution are imbalanced, resulting uniform placement of RSUs not the optimal scheme providing the maximum connectivity; On the other hand, connectivity of the communication in hot-spot road segments is well in general, especially when the vehicle density is high. Placing too many RSUs in the hop-spot road segments will not gain a remarkable improvement of connectivity, but decrease the connectivity for vehicles in other road segments. 


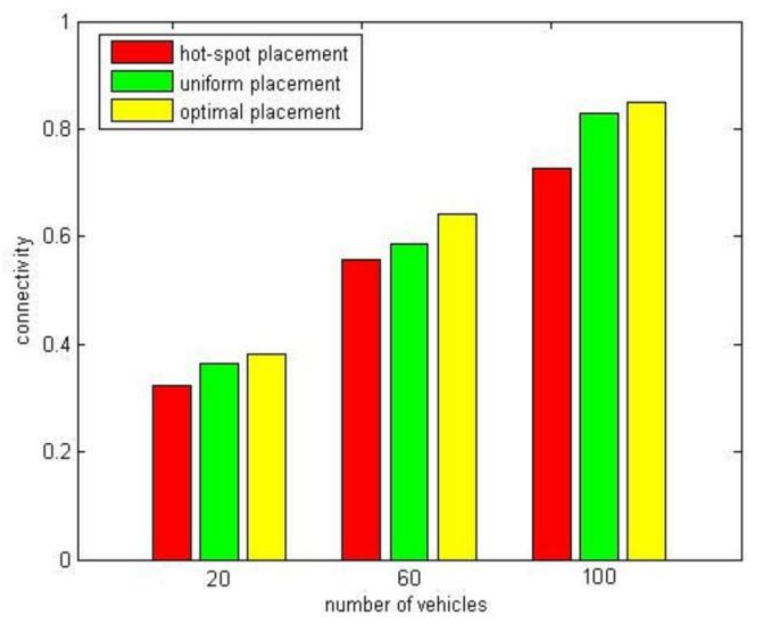

Figure 9. Simulation results

The rationality of the calculated results is verifie by taking a simulation using NS-2. We select the optimal placement schemes (C4, C6, C16, C19), (C3, C9, C16, C19) and (C3, C9, $\mathrm{C} 16, \mathrm{C} 20)$ when the number of vehicles is 20,60 and 100 respectively. Through multiple simulation experiments, statistical connectivty probabilities for the three kinds of schemes, under different numbers of vehicles, are shown in Fig. 9. From the results we can see that they agrees with the tendency of the calculated result The connectivity probability increases as the growth of vehicles. The optimal placement scheme performs better than the uniform placement and the hot-spot placement. Results shown in Figure.9 verify the rationality of our RSU optimal placement scheme.

\section{Conclusions}

In this paper, we study the optimal RSUs placement scheme in urban scenario, of which the spatial distribution of Vehicles is imbalanced. Our objective is to find an optimal position combination for placing a certain number of RSUs, so that the connectivity could be improved. We formulate the RSUs placement problem as a combinatorial optimization problem in the wo-dimensional space. The optimal objective function is the average connectivity probability. We test and verify our optimal placement strategy under different numbers of vehicles in an actual urban road network. Compared to the uniform placement and the hot-spot placement, the proposed optimal placement scheme performs better in connectivity. The simulation results validate the rationality of the strategy. In further work, we will extend for a more complex road topology and some other optimum indexes to further ascertain the effectiveness and rationality of our proposed optimization strategy.

\section{References}

[1] C. Lochert, B. Scheuermann, C. Wewetzer, A. Luebke and M. Mauve, "Data aggregation and roadside unit placement for a VANET traffic information system", Proceedings of the 5th ACM International Workshop on VehiculAr Inter-NETworking, San Francisco, USA, (2008), pp. 58-65. 
[2] F. Zou, J. F. Zhong, W. L. Wu, D. Z. Du and J. Lee, "Energy-efficient roadside unit scheduling for maintaining connectivity in vehicle ad-hoc network", Proceedings of the 5th International Conference on Ubiquitous Information Management and Communication, New York, USA, (2011).

[3] K. Mershad, H. Artail and M. Gerla, "We can deliver messages to far vehicles", IEEE Transactions on Intelligent Transportation Systems, vol.13, no. 3, (2012), pp. 1099-1115.

[4] A. Abdrabou, B. Liang and W. H. Zhang, "Delay analysis for a reliable message delivery in sparse vehicular ad hoc networks. Proceedings of Global Telecommunications Conference, Miami, USA, (2010) December 610 , pp. 1-5.

[5] Y. S. Liang, H. Liu and D. Rajan, "Optimal placement and configuration of roadside units in vehicular networks", Proceedings of the 2012", IEEE 75th Vehicular Technology Conference (VTC Spring), Yokohama, Japan, (2012) May 6-9, pp. 1-6.

[6] Y. Zhang, J. Zhao and G. Cao, "Service scheduling of vehicle-roadside data access", Mobile Networks and Applications, vol. 15, no. 1, (2010), pp. 83-96.

[7] A. Bohm and M. Jonsson, "Handover in IEEE 802.11p-based delay-sensitive vehicle-to-infrastructure Communication”, Technical Report IDE-0924, Halmstad University, Sweden, (2009).

[8] T. J. Wu, W. J. Liao and C. J. Chang, "A cost-effective strategy for road-side unit placement in vehicular network", IEEE Transactions on Communications, vol. 60, no. 8, (2012), pp. 2295-2303.

[9] B. B. Dubey, N. Chauhan and S. Pant, "Effect of position of fixed infrastructure on data dissemination in VANET", International Journal of Research and Reviews in Computer Science (JRRCS), vol. 2, no. 2, (2011), pp. 482-486.

[10] M. Rebai, L. Khoukhi, H. Snoussi and F. Hnaien, "Optimal placement in hybrid VANET-sensors networks", Wireless Advanced (WiAd), (2012), pp. 54-57.

[11] F. Malandrino, C. Casetti, C. F. Chinasserini and M. Fiore, "Content downloading in vehicular networks: what really matters", 2011 Proceedings IEEE INFOCOM, Shanghai, China, (201) April 10-15, pp. 426-430.

[12] A. Abdrabou and W. H. Zhuang, "Probabilistic delay control and roadside unit placement for vehicular ad hoc networks with disrupted connectivity", IEEE Journal on Selected Areas in Communications, vol. 29, no. 1, (2011), pp. 129-139.

[13]B. Aslam, F. Amjad and C. C. Zou, "Optimal roadside units pla ement in urban areas for vehicular networks", IEEE Symposium on Computers and Communications (ISCC), Cappadocia, Turkey, (2012) July 1-4, pp. 423-429.

[14] B. Aslam and C. C. Zou, "Optimal roadside units placement along highway", IEEE Consumer Communications and Networking Conference (CENC), Das Vegas, USA, (2011) January 9-12, pp. 814-815.

[15] J. Lee, C. M. Kim, "A roadside unit placement scheme for vehicular telematics networks", Advances in Computer Science and Information Technology, vol. 6059, (2010), pp. 196-202.

[16]Q. Yang, A. Lim, S. Li, JFang and P. Agrawal "ACAR: Adaptive connectivity aware routing for vehicular ad hoc networks in city scenarios", Mobile Network Apl., vol. 15, (2010), pp. 36-60.

[17]Q. Yang, A. Lim, S. Li, J. Fang and P Aglawal, "ACAR: Adaptive connectivity aware routing protocol for vehicular ad hoc networks", Proceeding of 17th International Conference on Computer Communication and Networks, St. Thomas, US Virgin Islands, (2008) August 3-7, pp. 1-6.

[18]Q. Yang, A. Lim and P. Agrawal, "Connectivity aware routing in vehicular networks", Wireless Communications and Net rorking Conferences, Las Vegas, USA, (2008) March 31-April 3, pp. 2218-2223.

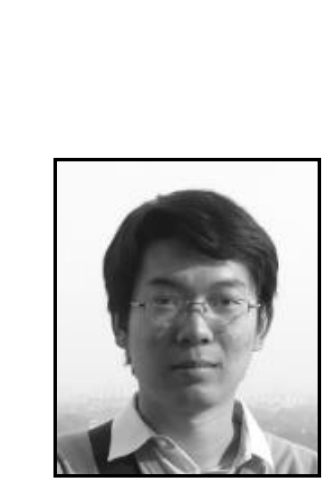

\section{Authors}

\section{Liu Hai-qing}

He is currently enrolled for joint courses for master and doctor degrees in School of Control Science and Engineering, Shandong University. His research interests include intelligent transportation system, vehicular ad hoc network and cooperative vehicle infrastructure system. 


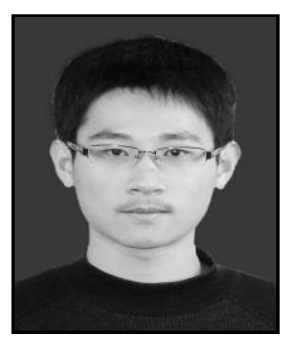

\section{Yang Tai}

He has a major in Communication Engineering, third year student in School of Information Science and Engineering Science, Shandong University. His research interest is communication technology and applications.

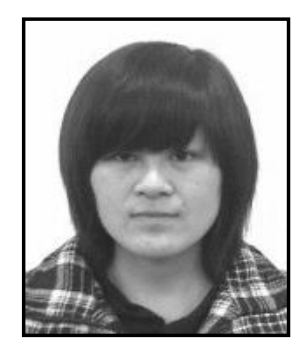

\section{Ding Si-jing}

She is a graduate student of School of Control Science/and Engineering, Shandong University, majoring in Control Scjence and Engineering. Her research interests include intelligent transportation system and vehicular ad hoc network.

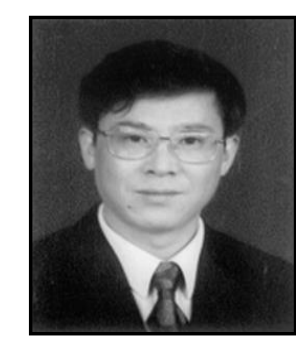

\section{Yang Li-cai}

He is currently a professor and doetoral supervisor at School of Control Science and Engineering in Shandong University. His research interests include artificlal intelligence and intelligent control, control theory and applications, intelligent transportation systems, and vehicular ad hoc networks.

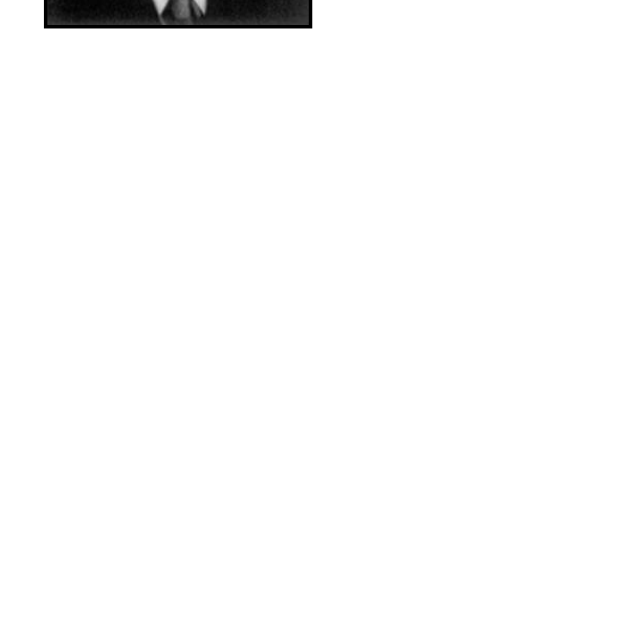

